\begin{tabular}{|c|c|c|c|c|}
\hline Caucasian Journal of Science & ISSN: $2148-6840$ \\
\hline https://doi.org/10.48138/cjo.972212 & $\begin{array}{c}\text { Year: } 2021 \\
\text { Volume: } 8 \text { Issue: } 2\end{array}$
\end{tabular}

\title{
(4-Carbamoylphenyl)Boronic Acid: A DFT Study On The Structural And Spectral Properties
}

\author{
Ahmet KUNDURACIOĞLU1
}

Makalenin Alanı: Kimya

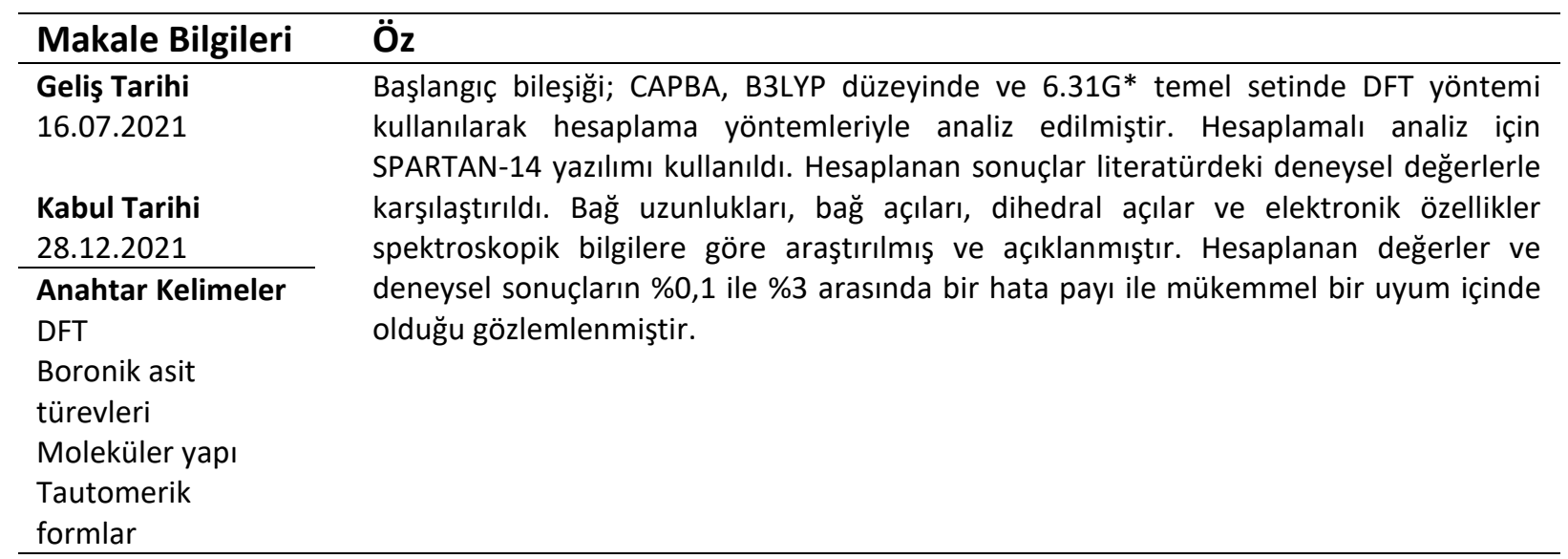

\begin{tabular}{ll}
\hline Article Info & Abstract \\
\hline $\begin{array}{l}\text { Received } \\
\text { 16.07.2021 }\end{array}$ & $\begin{array}{l}\text { Title compound; CAPBA was analyzed via computational methods using the DFT method } \\
\text { in the B3LYP level and 6.31G* basis set. The SPARTAN-14 software was used for } \\
\text { computational analysis. The calculated results were compared to experimental values } \\
\text { from the literature. Bond lengths, bond angles, dihedral angles and electronic } \\
\text { Accepted }\end{array}$ \\
$\begin{array}{l}\text { properties were investigated and explicated according to spectroscopic knowledge. The } \\
\text { calculated values and experimental results were observed to be in perfect agreement } \\
\text { Keywords } \\
\text { DFT }\end{array}$ & $\begin{array}{l}\text { in an error margin between 0,1\% and 3\%. } \\
\text { Boronic acid } \\
\text { derivatives, } \\
\text { Molecular } \\
\text { structure, }\end{array}$ \\
Tautomeric forms & \\
\hline
\end{tabular}

\section{INTRODUCTION}

Boronic acid and its derivatives (BAs) are well known for their wide use in, especially medicinal chemistry. Their chemical and biological activities depend upon their additional substituents (Hall, 2006, pp. 1-99). BAs exhibit Lewis acid properties due to the lack of $\mathrm{e}^{-}$in

\footnotetext{
${ }^{1}$ Bursa Uludağ Üniversitesi Mustafa Kemal Paşa MYO-Bursa; e-mail: akunduracioglu@uludag.edu.tr; ORCID: 0000-0002-4421-9912 (Sorumlu yazar)
} 
the valence shell of the Boron atom. Boron atom attracts a $\mathrm{OH}^{-}$anion from water in aqueous solutions to fill its valence shell (Zepeda-Valesquez, 2015, pp.6-7)

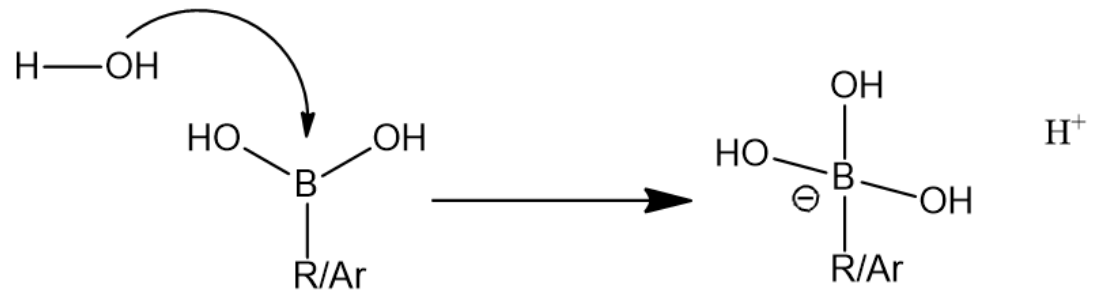

Figure 1. Acidic behavior of boronic acid in aqueous solution.

Previously, all boron-containing compounds were assumed to be poisonous, and their use was limited except for some ant poisoning chemicals. But recently, this prejudice has been broken and boron compounds have been acquitted in this regard, so their use in pharmaceutical chemistry has been paved (Baker et al., 2009). Because of their importance in chemical and medicinal studies such as Boron Neutron Capture Therapy (BNCT) (Tjarks et al.,1992) preparing medicines (Kar, 2003, pp.143-146), etc. a huge number of studies has been undergone about spectroscopic and structural properties of the organoboron compounds particularly on boronic acids and their esters. These studies generally survey the molecular structures as a dimer or monomer-dimer equilibrium Lu et al., Zheng et al., Rani et al., Karabacak et al., 2012, Zheng et al., 2011, Sachan et al., 2014, Erdoğdu et al., 2009).<smiles>OB(O)O</smiles>

boric acid<smiles>[R]OB([R])[R]</smiles>
boronic ester<smiles>[R]B([R])[R]</smiles>

Borane<smiles>[R]B1OB([R])OB([R])O1</smiles>
(Cyclic boronic anhydride)

$\mathrm{R}, \mathrm{R}^{\prime}, \mathrm{R}^{\prime \prime}=$ alkyl or aryl<smiles>[R]B(O)O</smiles>

boronic acid<smiles>[R]B([R])O</smiles>

borinic acid

Figure 2. Oxygen-containing organoboron compounds (Hall 2006) 
In some recent studies, researchers have handled their effect on the formation of biofilm, production of virulence factors (elastase and pyocyanin) and swarming motility in Pseudomonas aeruginosa (Akalın\&Ulusoy, 2018).

CAPBA is a white to Brown solid with a melting point of $229-234^{\circ} \mathrm{C}$ (URL1). Apostolova et al. experimentally found the molecular properties of the title compound 4Carbamoylphenylboronic acid (AKA 4-Aminocarbonylphenylboronic acid / CAPBA) in a crystallographic study (Apostolova et al., 2010). But unfortunately, there is no other experimental spectral data for this compound so far.

\section{EXPERIMENTAL}

\section{Computational Details}

In all calculations, the SPARTAN 14 computational chemistry suite (Hehre, 2014) was used. Calculations were undergone according to the DFT method in the B3LYP level and 6.31G* basis set (Becke, 1993, Hehre, 2014, Jensen, 2017, Onishi, 2018, Peter et al., 2011, Silverstein et al., 2005). The calculated and experimental results were tabulated in relational tables for comparisons. Recently, DFT method has gained a rising popularity among the researchers. Most researchers especially the ones investigating d-block metal complexes prefer to use this method with or instead of HF. (Beytur\&Yuksek, 2018). Some small molecules such as boronic acid derivatives or benzoic acid derivatives are investigated using both DFT and HF and mostly In many studies, it was seen that the results of the DFT method were more compatible with the experimental results than the HF method (Kotan\&Yüksek, 2019).

The DFT method has also been successfully used in the study of other small groups of molecules such as pyrazoles and their derivatives (Turhan İrak\&Beytur 2019).

Molecular Structure CAPBA has a dimeric structure like other boronic acid derivatives which has been investigated in depth so far. But in these studies, CAPBA never was handled for its tautomeric forms. Due to the lack of a survey on its tautomers, in the following study, it has been handled considering its tautomeric forms and conformational isomers on the same hand. The dimeric structure was omitted for clarity (Figure $3 \mathrm{a}$ and b). The group of $-\mathrm{B}(\mathrm{OH})_{2}$ has four conformers which will be abbreviated as "CC", "CT", "TC" and " $\mathrm{TT}$ " in the following pages $(\mathrm{C}=\mathrm{Cis}, \mathrm{T}=$ Trans $)$. 

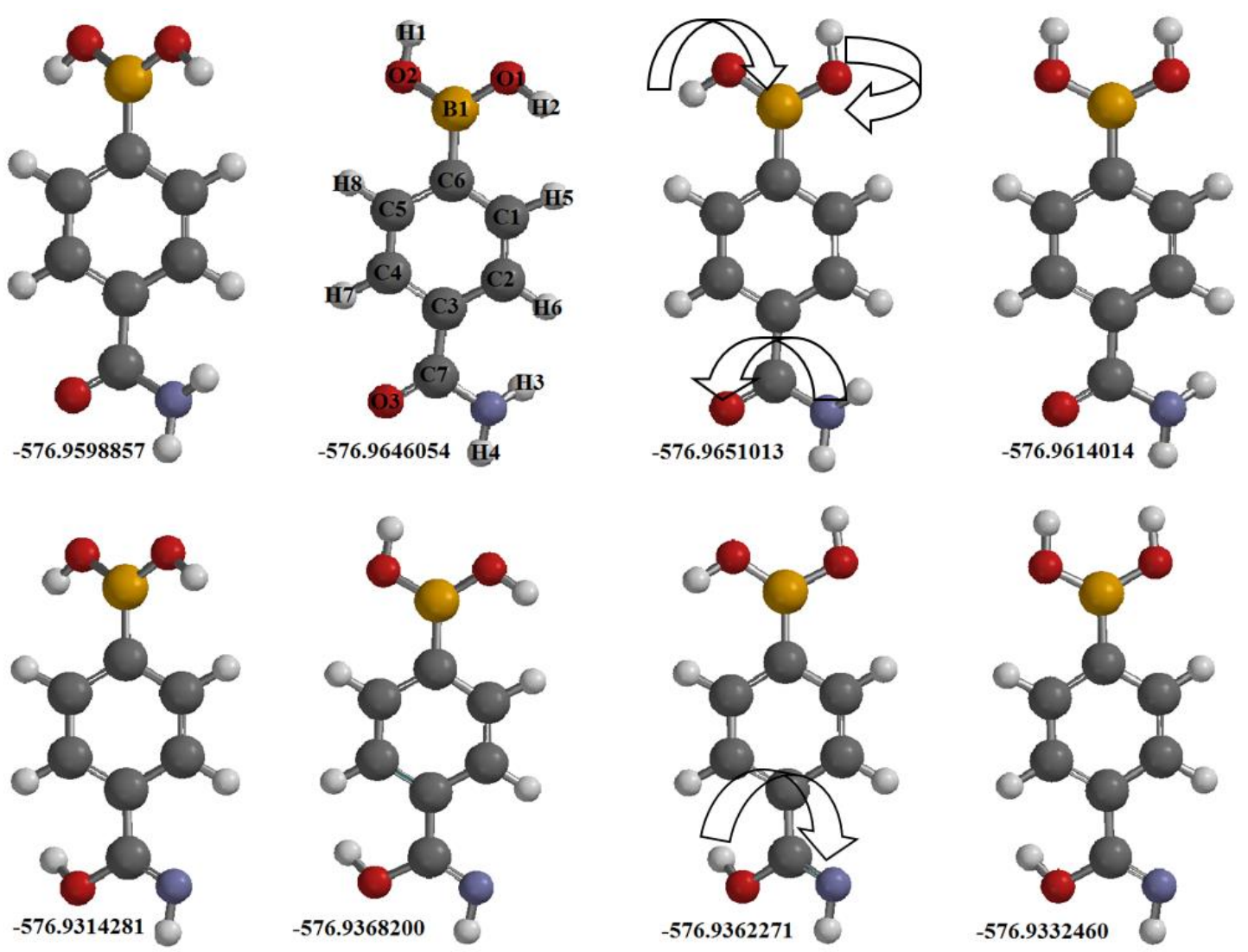

Figure 3. The isomeric forms and tautomeric transformation of the compound CAPBA (energies in Hartree)

\section{Molecular Structure}

As mentioned before, CAPBA had been investigated for its crystallographical structure via single-crystal X-ray spectroscopy (Apostolova et al., 2010). Both the experimental and the calculated bond lengths and bond angles have been tabulated in Table 1 and Table 2 respectively. Besides calculated dihedral angles have been given in Table 3 for gaining an idea about the torsion of some parts in the molecule. Unfortunately, experimental dihedral angles are not available in the literature.

As seen in Table 1, the calculated bond lengths are very close to the experimentally measured ones. Since the least energetic form of the compound is TC the error\% values were calculated between the experimental values and the ones that belong to TC. 
Table 1. Bond distances (Á) for the compound CAPBA

\begin{tabular}{|c|c|c|c|c|c|c|c|c|c|c|}
\hline Distance (Ấ) & CC1 & CC2 & СТ1 & CT2 & TC1 & TC2 & TT1 & TT2 & EXP* & Error\% \\
\hline $\mathrm{H3}, \mathrm{O3}$ & 3.149 & 0.971 & 3.145 & 0.971 & 3.149 & 0.971 & 3.145 & 0.971 & $x x$ & $x$ \\
\hline $\mathrm{H} 4, \mathrm{O3}$ & 2.460 & 2.354 & 2.457 & 2.351 & 2.459 & 2.353 & 2.456 & 2.349 & $x x$ & $x$ \\
\hline $\mathrm{O} 1, \mathrm{H} 2$ & 0.966 & 0.966 & 0.966 & 0.966 & 0.970 & 0.970 & 0.967 & 0.967 & 0.820 & 18.29 \\
\hline $02, \mathrm{H} 1$ & 0.966 & 0.966 & 0.970 & 0.970 & 0.967 & 0.966 & 0.967 & 0.967 & 0.820 & 17.93 \\
\hline $\mathrm{H} 4, \mathrm{~N} 1$ & 1.012 & 1.024 & 1.012 & 1.024 & 1.012 & 1.024 & 1.012 & 1.024 & 0.860 & 17.67 \\
\hline H3,N1 & 1.010 & 3.084 & 1.010 & 3.085 & 1.010 & 3.083 & 1.010 & 3.084 & 0.860 & 17.44 \\
\hline $\mathrm{N} 1, \mathrm{C7}$ & 1.376 & 1.271 & 1.378 & 1.272 & 1.376 & 1.271 & 1.378 & 1.272 & 1.298 & 6.01 \\
\hline $\mathrm{O}, \mathrm{C7}$ & 1.224 & 1.371 & 1.224 & 1.371 & 1.225 & 1.372 & 1.225 & 1.372 & 1.246 & -1.69 \\
\hline $\mathrm{C} 7, \mathrm{C3}$ & 1.504 & 1.490 & 1.503 & 1.489 & 1.503 & 1.489 & 1.503 & 1.489 & 1.505 & -0.13 \\
\hline $\mathrm{C} 2, \mathrm{C} 1$ & 1.394 & 1.390 & 1.393 & 1.390 & 1.393 & 1.390 & 1.393 & 1.390 & 1.384 & 0.65 \\
\hline $\mathrm{C} 3, \mathrm{C2}$ & 1.401 & 1.403 & 1.402 & 1.402 & 1.402 & 1.403 & 1.402 & 1.403 & 1.397 & 0.36 \\
\hline $\mathrm{C} 4, \mathrm{C} 3$ & 1.401 & 1.402 & 1.401 & 1.403 & 1.400 & 1.402 & 1.401 & 1.403 & 1.388 & 0.86 \\
\hline C5,C4 & 1.391 & 1.394 & 1.391 & 1.393 & 1.391 & 1.394 & 1.391 & 1.394 & 1.384 & 0.51 \\
\hline $\mathrm{C} 6, \mathrm{C} 5$ & 1.407 & 1.404 & 1.407 & 1.405 & 1.406 & 1.404 & 1.407 & 1.404 & 1.391 & 1.08 \\
\hline C1,C6 & 1.406 & 1.408 & 1.405 & 1.407 & 1.406 & 1.409 & 1.406 & 1.408 & 1.391 & 1.08 \\
\hline B1,01 & 1.368 & 1.367 & 1.374 & 1.374 & 1.367 & 1.366 & 1.375 & 1.374 & 1.351 & 1.18 \\
\hline B1,02 & 1.367 & 1.368 & 1.366 & 1.367 & 1.374 & 1.374 & 1.374 & 1.374 & 1.393 & -1.36 \\
\hline $\mathrm{C} 6, \mathrm{~B} 1$ & 1.580 & 1.580 & 1.571 & 1.571 & 1.571 & 1.571 & 1.564 & 1.564 & 1.546 & 1.62 \\
\hline $\mathrm{H} 6, \mathrm{C} 2$ & 1.087 & 1.085 & 1.087 & 1.085 & 1.087 & 1.085 & 1.087 & 1.085 & 0.930 & 16.88 \\
\hline $\mathrm{H} 5, \mathrm{C} 1$ & 1.089 & 1.089 & 1.090 & 1.089 & 1.086 & 1.086 & 1.086 & 1.086 & 0.930 & 16.77 \\
\hline $\mathrm{H7}, \mathrm{C4}$ & 1.085 & 1.088 & 1.085 & 1.088 & 1.085 & 1.088 & 1.085 & 1.088 & 0.930 & 16.67 \\
\hline $\mathrm{H} 8, \mathrm{C} 5$ & 1.089 & 1.089 & 1.086 & 1.086 & 1.090 & 1.089 & 1.086 & 1.086 & 0.930 & 17.20 \\
\hline
\end{tabular}

As seen in the table, the bonds between $\mathrm{C}$ and $\mathrm{C}$ or $\mathrm{C}$ and Heteroatom were calculated with small deviations which are between $0.36 \%$ and $6 \%$, from the experimental values. But the bonds between $\mathrm{H}$ and $\mathrm{C}$ or $\mathrm{H}$ and heteroatoms were calculated with deviations between $16 \%$ to $18 \%$. Also, another point to notice, most values are very close to each other despite the tautomeric activity except for the $\mathrm{H} 3-\mathrm{O} 3$ bond which changes dramatically from one form to another due to $\mathrm{H} 3 \mathrm{immigration}$ between N1 to O3. As the last word for bond distances, between two tautomers for every isomer, the bond distances change slightly between 0 to 2

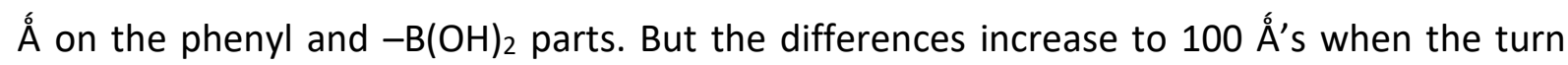
comes to bonds between N1, C7 and $\mathrm{O} 3$. 
The bond angles for the compound have been tabulated in table 2. According to these values, the most mobile parts of the molecule are again the group of $-\mathrm{B}(\mathrm{OH})_{2}$ and carbamoyl part.

Table 2. Some bond angles $\left({ }^{\circ}\right)$ for the compound CAPBA

\begin{tabular}{|c|c|c|c|c|c|c|c|c|c|c|}
\hline Angle $\left({ }^{\circ}\right)$ & CC1 & CC2 & CT1 & CT2 & TC1 & TC2 & TT1 & TT2 & EXP* & err\% \\
\hline $\mathrm{H} 3,03, \mathrm{C7}$ & 22.92 & 109.71 & 23.06 & 109.65 & 22.94 & 109.55 & 23.08 & 109.45 & $x x$ & $\mathrm{x}$ \\
\hline O3,C7,N1 & 121.86 & 121.85 & 121.78 & 121.75 & 121.77 & 121.80 & 121.61 & 121.67 & 120.80 & 0.80 \\
\hline $\mathrm{C7}, \mathrm{N} 1, \mathrm{H} 4$ & 115.13 & 110.00 & 114.90 & 109.92 & 115.12 & 109.94 & 114.94 & 109.84 & 120.00 & 4.07 \\
\hline $\mathrm{H} 3, \mathrm{N1}, \mathrm{C7}$ & 120.23 & 19.13 & 119.88 & 19.06 & 120.23 & 19.15 & 119.93 & 19.03 & 120.00 & 0.19 \\
\hline $\mathrm{H} 4, \mathrm{~N} 1, \mathrm{H} 3$ & 116.32 & 91.25 & 116.11 & 91.20 & 116.36 & 91.18 & 116.23 & 91.14 & 120.00 & 3.03 \\
\hline $\mathrm{N} 1, \mathrm{C} 7, \mathrm{C} 3$ & 116.24 & 121.26 & 116.16 & 121.34 & 116.26 & 121.40 & 116.19 & 121.56 & 118.80 & 2.14 \\
\hline $\mathrm{O}, \mathrm{C7}, \mathrm{C3}$ & 121.88 & 116.89 & 122.05 & 116.90 & 121.96 & 116.79 & 122.18 & 116.76 & 120.40 & 1.30 \\
\hline $\mathrm{C7}, \mathrm{C3}, \mathrm{C2}$ & 123.52 & 119.29 & 123.28 & 119.21 & 123.58 & 119.40 & 123.33 & 119.37 & 122.20 & 1.13 \\
\hline $\mathrm{C} 3, \mathrm{C} 2, \mathrm{C} 1$ & 120.17 & 120.17 & 120.03 & 120.03 & 120.30 & 120.29 & 120.19 & 120.21 & 121.10 & 0.66 \\
\hline$C 4, C 3, C 2$ & 119.06 & 118.91 & 119.11 & 118.91 & 119.08 & 118.93 & 119.15 & 118.96 & 117.80 & 1.09 \\
\hline $\mathrm{C5}, \mathrm{C4}, \mathrm{C3}$ & 120.25 & 120.34 & 120.36 & 120.49 & 120.12 & 120.22 & 120.30 & 120.41 & 121.20 & 0.89 \\
\hline $\mathrm{C6}, \mathrm{C5}, \mathrm{C4}$ & 121.59 & 121.54 & 121.36 & 121.30 & 121.68 & 121.60 & 121.24 & 121.20 & 120.80 & 0.73 \\
\hline C1,C6,C5 & 117.35 & 117.25 & 117.49 & 117.37 & 117.46 & 117.37 & 117.85 & 117.74 & 118.20 & 0.63 \\
\hline $\mathrm{C} 2, \mathrm{C} 1, \mathrm{C} 6$ & 121.57 & 121.78 & 121.63 & 121.88 & 121.35 & 121.58 & 121.26 & 121.47 & 120.80 & 0.46 \\
\hline B1,01,H2 & 111.96 & 112.14 & 113.16 & 113.29 & 110.65 & 110.76 & 114.84 & 114.96 & $x x$ & $\mathrm{x}$ \\
\hline $\mathrm{B} 1,02, \mathrm{H} 1$ & 112.05 & 112.04 & 110.73 & 110.69 & 113.22 & 113.27 & 114.92 & 114.87 & $x x$ & $x$ \\
\hline C6,B1,O2 & 122.22 & 122.17 & 118.41 & 118.38 & 123.83 & 123.92 & 118.01 & 117.94 & 121.60 & 1.83 \\
\hline C6,B1,01 & 122.15 & 122.21 & 123.87 & 123.85 & 118.43 & 118.34 & 117.98 & 117.97 & 119.40 & 0.81 \\
\hline C1,C6,B1 & 121.25 & 121.44 & 122.20 & 122.40 & 120.21 & 120.32 & 120.98 & 121.23 & 119.50 & 0.59 \\
\hline C5,C6,B1 & 121.40 & 121.30 & 120.31 & 120.23 & 122.32 & 122.31 & 121.16 & 121.03 & 122.20 & 0.10 \\
\hline $\mathrm{H} 6, \mathrm{C} 2, \mathrm{C} 1$ & 119.39 & 121.38 & 119.55 & 121.44 & 119.32 & 121.27 & 119.43 & 121.32 & 119.50 & 0.15 \\
\hline $\mathrm{H} 5, \mathrm{C} 1, \mathrm{C} 6$ & 119.67 & 119.57 & 120.21 & 120.08 & 119.00 & 118.91 & 119.22 & 119.11 & 119.60 & 0.50 \\
\hline $\mathrm{C2}, \mathrm{C} 1, \mathrm{H} 5$ & 118.74 & 118.63 & 118.15 & 118.02 & 119.64 & 119.51 & 119.52 & 119.42 & 119.60 & 0.03 \\
\hline $\mathrm{H7}, \mathrm{C} 4, \mathrm{C} 5$ & 121.45 & 119.15 & 121.32 & 119.02 & 121.52 & 119.20 & 121.39 & 119.09 & 119.40 & 1.78 \\
\hline $\mathrm{C} 3, \mathrm{C} 2, \mathrm{H} 6$ & 120.39 & 118.45 & 120.39 & 118.53 & 120.34 & 118.44 & 120.33 & 118.46 & 119.50 & 0.70 \\
\hline $\mathrm{H} 8, \mathrm{C} 5, \mathrm{C} 4$ & 118.79 & 118.69 & 119.66 & 119.60 & 118.16 & 118.07 & 119.57 & 119.48 & $x x$ & $\mathrm{x}$ \\
\hline 01,B1,02 & 115.63 & 115.62 & 117.73 & 117.77 & 117.73 & 117.74 & 124.02 & 124.09 & 118.90 & 0.98 \\
\hline $\mathrm{C2}, \mathrm{C3}, \mathrm{C7}$ & 123.52 & 119.29 & 123.28 & 119.21 & 123.58 & 119.40 & 123.33 & 119.37 & $x x$ & $x$ \\
\hline $\mathrm{C7}, \mathrm{C3}, \mathrm{C4}$ & 117.41 & 121.80 & 117.60 & 121.88 & 117.34 & 121.67 & 117.52 & 121.67 & 120.00 & 2.22 \\
\hline $\mathrm{H7}, \mathrm{C} 4, \mathrm{C} 3$ & 118.30 & 120.46 & 118.32 & 120.44 & 118.37 & 120.53 & 118.31 & 120.45 & 119.40 & 0.86 \\
\hline $\mathrm{C} 6, \mathrm{C} 5, \mathrm{H} 8$ & 119.62 & 119.75 & 118.97 & 119.09 & 120.15 & 120.32 & 119.18 & 119.31 & 119.60 & 0.46 \\
\hline
\end{tabular}


As seen from Table 2 the error margin is between $0.03 \%$ and $4 \%$. An important point to be noticed from the table, there is a $5-6^{\circ}$ difference between the tautomeric forms when it comes to carbamoyl groups such as N1, C7, C3 and O3, C7, C3 angles. But in the phenyl part and $-\mathrm{B}(\mathrm{OH})_{2}$ group, the values only change slightly. For example for $\mathrm{C} 6, \mathrm{~B} 1, \mathrm{O} 2$ the values are $123.83^{\circ}$ and $123.92^{\circ}$, for $\mathrm{O} 1, \mathrm{~B} 1, \mathrm{O} 2$ angle $117.73^{\circ}$ and $117.74^{\circ}$ between two tautomers for TC isomer.

In the literature, there is no data for the torsional properties of CAPBA, for this reason, Table 3 has no experimental values column. Since the values in Table1 and Table 2 are close to the experimental results enough, it can be considered that Table 3 is also reliable.

As seen in Table 3 torsion angles in the CAPBA molecule differs according to the sections. In the phenyl part, as expected, for example, dihedral angles are about 1-2 degrees. For instant $\mathrm{H} 6, \mathrm{C} 2, \mathrm{C} 1, \mathrm{H} 5-1.93 / 0.64^{\circ}$ for $\mathrm{TC}$ isomer and $\mathrm{C} 1, \mathrm{C} 2, \mathrm{C} 3, \mathrm{C} 4$ has $0.78 /-0.93^{\circ}$. But $\mathrm{O} 3, \mathrm{C} 7, \mathrm{C} 3, \mathrm{C} 2$ and $\mathrm{N} 1, \mathrm{C} 7, \mathrm{C} 3, \mathrm{C} 4$ has $160.83^{\circ} / 157.24^{\circ}$ and $-162.87^{\circ} /-155.72^{\circ}$ respectively.

Table 3. Dihedral angles for CAPBA and conformers.

\begin{tabular}{|c|c|c|c|c|c|c|c|c|}
\hline Dihedral & CC1 & $\mathrm{CC2}$ & CT1 & CT2 & TC1 & TC2 & TT1 & TT2 \\
\hline H3,O3,N1,H4 & 135.70 & -155.87 & -134.05 & -157.00 & 135.86 & -155.61 & -134.62 & -157.49 \\
\hline H3,03,C7,C3 & -165.14 & 16.94 & 164.54 & 16.02 & -165.26 & 17.05 & 164.79 & 15.61 \\
\hline $\mathrm{H} 4, \mathrm{~N} 1, \mathrm{C7}, \mathrm{O3}$ & -9.66 & 2.41 & 10.09 & 2.33 & -9.83 & 2.47 & 10.23 & 2.30 \\
\hline $\mathrm{H} 3, \mathrm{~N} 1, \mathrm{C7}, \mathrm{O3}$ & -156.81 & 14.32 & 155.79 & 13.65 & -157.05 & 14.49 & 156.36 & 13.42 \\
\hline $\mathrm{H} 4, \mathrm{~N} 1, \mathrm{C} 7, \mathrm{C} 3$ & 171.67 & -178.70 & -171.31 & -178.65 & 171.45 & -178.57 & -171.02 & -178.58 \\
\hline $\mathrm{H} 3, \mathrm{~N} 1, \mathrm{C7}, \mathrm{C3}$ & 24.53 & -166.79 & -25.61 & -167.33 & 24.22 & -166.55 & -24.89 & -167.46 \\
\hline H3,03,C7,N1 & 16.27 & -164.12 & -16.95 & -164.92 & 16.09 & -163.94 & -16.53 & -165.23 \\
\hline $\mathrm{H} 1,02,01, \mathrm{H} 2$ & -20.84 & -21.27 & 170.00 & 171.95 & 172.16 & -170.16 & -0.08 & 0.12 \\
\hline H1,02,B1,01 & 173.43 & 173.23 & -1.88 & -1.49 & 175.90 & -174.84 & -0.24 & -0.13 \\
\hline H2,01,B1,O2 & 173.36 & 173.30 & 174.76 & 175.78 & -1.46 & 1.82 & 0.15 & 0.26 \\
\hline C5,C6,B1,01 & 147.07 & 146.74 & 163.07 & 164.96 & 166.06 & -164.50 & 179.26 & -179.71 \\
\hline C5,C6,B1,O2 & -32.94 & -33.22 & -16.70 & -14.68 & -14.26 & 15.73 & -0.82 & 0.24 \\
\hline C1,C6,B1,01 & -33.42 & -32.85 & -16.47 & -14.50 & -14.55 & 15.82 & -0.36 & 0.61 \\
\hline $\mathrm{C} 3, \mathrm{C} 2, \mathrm{C} 1, \mathrm{C} 6$ & -0.39 & -0.06 & -0.45 & 0.18 & -0.26 & 0.85 & -0.17 & 0.42 \\
\hline $\mathrm{C} 3, \mathrm{C} 4, \mathrm{C} 5, \mathrm{C} 6$ & 0.23 & -0.43 & -1.21 & -0.38 & 0.48 & 0.32 & -0.73 & 0.04 \\
\hline $\mathrm{H7}, \mathrm{C} 4, \mathrm{C} 5, \mathrm{H} 8$ & 1.25 & -1.37 & -0.90 & -2.30 & 1.33 & -2.79 & -0.57 & -2.11 \\
\hline $\mathrm{B} 1, \mathrm{C} 6, \mathrm{C} 1, \mathrm{C} 2$ & -179.91 & -179.85 & -179.96 & 179.62 & -179.56 & 179.51 & -179.68 & -179.92 \\
\hline B1,C6,C1,H5 & -1.62 & -1.11 & -0.64 & -1.58 & -0.05 & -0.55 & 0.75 & -0.07 \\
\hline $\mathrm{B} 1, \mathrm{C} 6, \mathrm{C} 5, \mathrm{H} 8$ & -1.02 & -1.67 & 0.57 & -0.09 & -1.51 & 0.49 & 0.03 & -0.82 \\
\hline B1,C6,C5,C4 & 179.99 & -179.91 & -179.22 & -179.53 & 179.44 & 179.91 & -179.86 & 179.69 \\
\hline $\mathrm{H} 6, \mathrm{C} 2, \mathrm{C} 1, \mathrm{H} 5$ & -1.08 & 1.04 & 2.33 & 1.11 & -1.93 & 0.64 & 1.66 & 0.34 \\
\hline $\mathrm{C} 1, \mathrm{C} 6, \mathrm{~B} 1, \mathrm{O} 2$ & 146.56 & 147.19 & 163.75 & 165.86 & 165.12 & -163.96 & 179.56 & -179.44 \\
\hline $\mathrm{N} 1, \mathrm{C} 7, \mathrm{C} 3, \mathrm{C} 4$ & -162.43 & -156.40 & 161.28 & -157.32 & -162.87 & -155.72 & 162.34 & -156.67 \\
\hline $\mathrm{N} 1, \mathrm{C} 7, \mathrm{C} 3, \mathrm{C} 2$ & 18.66 & 22.73 & -19.56 & 22.10 & 17.90 & 23.75 & -18.43 & 22.76 \\
\hline $\mathrm{C} 1, \mathrm{C2}, \mathrm{C} 3, \mathrm{C} 4$ & 1.08 & -0.68 & -0.42 & -0.61 & 0.78 & -0.93 & -0.80 & -1.00 \\
\hline H1,02,B1,C6 & -6.56 & -6.80 & 177.91 & 178.18 & -3.78 & 4.94 & 179.85 & 179.92 \\
\hline H2,01,B1,C6 & -6.65 & -6.67 & -5.02 & -3.86 & 178.23 & -177.97 & -179.94 & -179.80 \\
\hline $\mathrm{O3}, \mathrm{C7}, \mathrm{C3}, \mathrm{C2}$ & -160.00 & -158.33 & 159.03 & -158.84 & -160.83 & -157.24 & 160.31 & -158.08 \\
\hline $\mathrm{O} 3, \mathrm{C} 7, \mathrm{C} 3, \mathrm{C} 4$ & 18.90 & 22.54 & -20.13 & 21.74 & 18.41 & 23.29 & -18.92 & 22.49 \\
\hline
\end{tabular}


Briefly, it can be said, the molecule is almost planar in the phenyl section and twisted about $15^{\circ}$ and $35^{\circ}$ in the substituted parts (Figure 4).

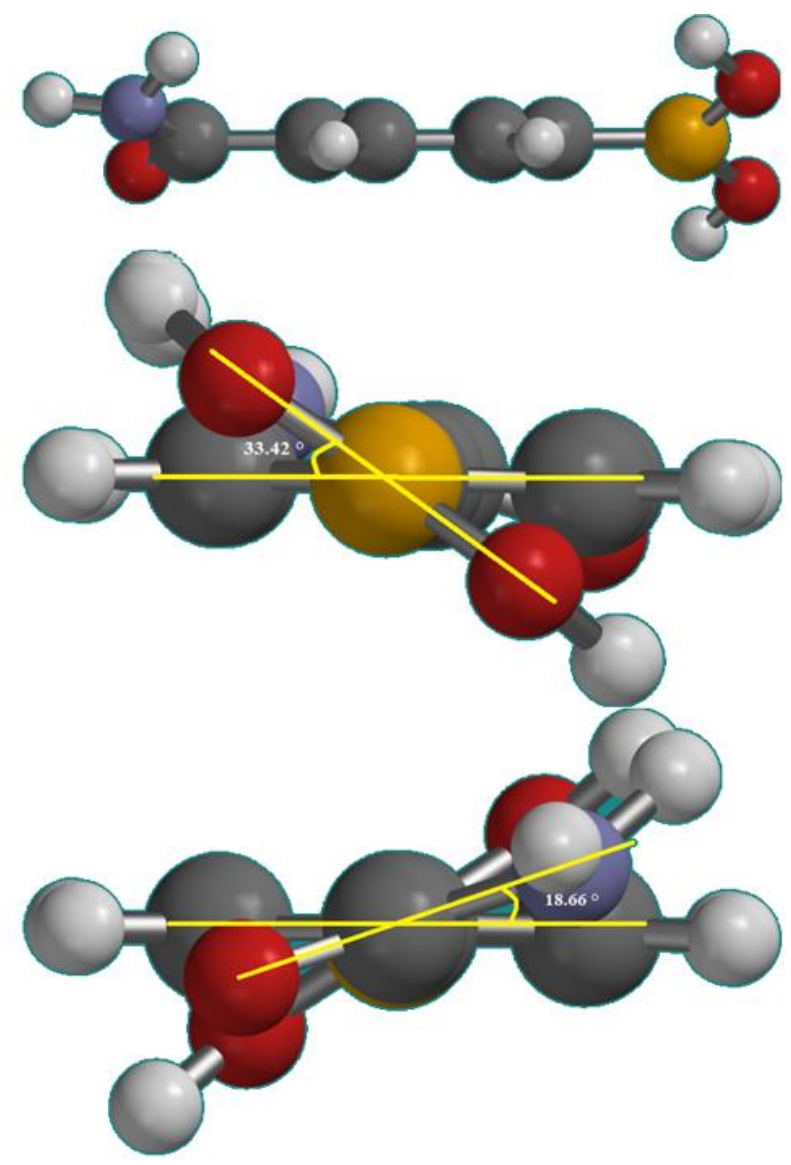

Figure 4. Deviation from planarity in parts of CAPBA

\section{Mulliken Charges Analysis}

Like every molecule, CAPBA's atoms have different electronegativities. For this reason, the electronic distribution on the molecule is not homogenous. The more an atom is electronegative, the more electrons it gathers up. This heterogeneity causes different partial charges on atoms of the same molecule. If a certain section of a molecule is electronically rich, it is a partially negative site and so on. Electron-rich parts of the molecules are more suitable for electrophilic attacks and electron-poor sites are open for nucleophilic attacks. Mulliken charge distribution is important for predicting the potential reaction mechanisms that the molecule is involved (Jensen, 2016). ESPMap surface determines the distance that a certain positive charge can interact with the molecule enough to form an attraction or repulsion so 
that they can have a bonding possibility for complexation reaction. Molecular ESP (Vr) is calculated via Eq. 2 (Peter et al., 2011).

$$
V r=\sum_{A} \frac{Z_{A}}{R_{A}-r}-\int \frac{\rho\left(r^{\prime}\right)}{\left(r^{\prime}-r\right)} d r^{\prime}
$$

Figure 5 and Table 4 exhibit the Mulliken charges of CAPBA which are also depicted in figure 2 as colors. At a glance the most remarkable points are briefly:

1- $\quad$ C atoms of the molecule are divided into two parts; $\mathrm{C} 1,2,4$ and 5 are negatively charged and $C 3,6$ and 7 are charged positively. $C 7$ has the highest positive charge and its charge dramatically changes between tautomeric forms.

2- $\quad$ All $\mathrm{H}$ atoms have positive charges and the most striking differences are seen on $\mathrm{H} 4$ and $\mathrm{H} 8$ according to tautomeric changes.

3- All $\mathrm{O}$ atoms have negative charges and according to tautomeric activity only the charge of $\mathrm{O} 3$ changes.

4- $\quad \mathrm{N}$ atom has a considerable negative charge and as expected it changes between tautomeric forms.

5- $\quad \mathrm{B} 1, \mathrm{O} 1, \mathrm{O} 2, \mathrm{H} 1, \mathrm{H} 2$ and $\mathrm{H} 3$ are not affected by tautomeric transformations.

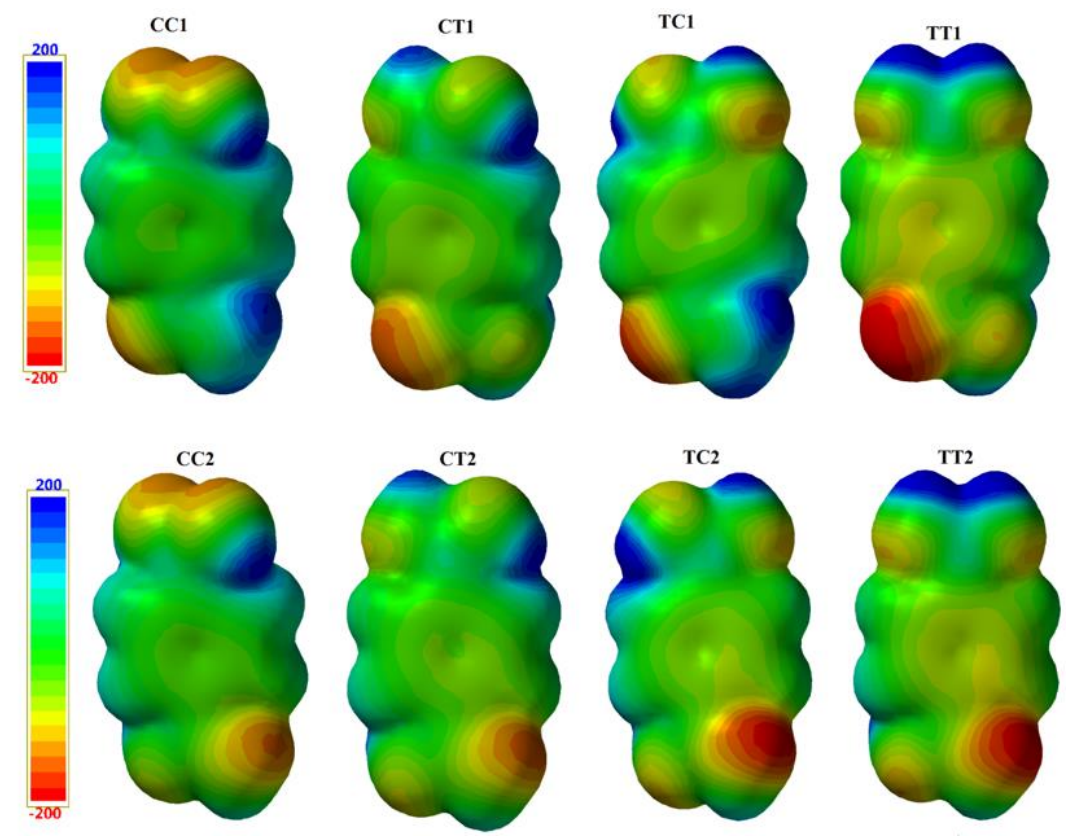

Figure 5. Mulliken Charge distribution of CAPBA according to isomeric and tautomeric forms 


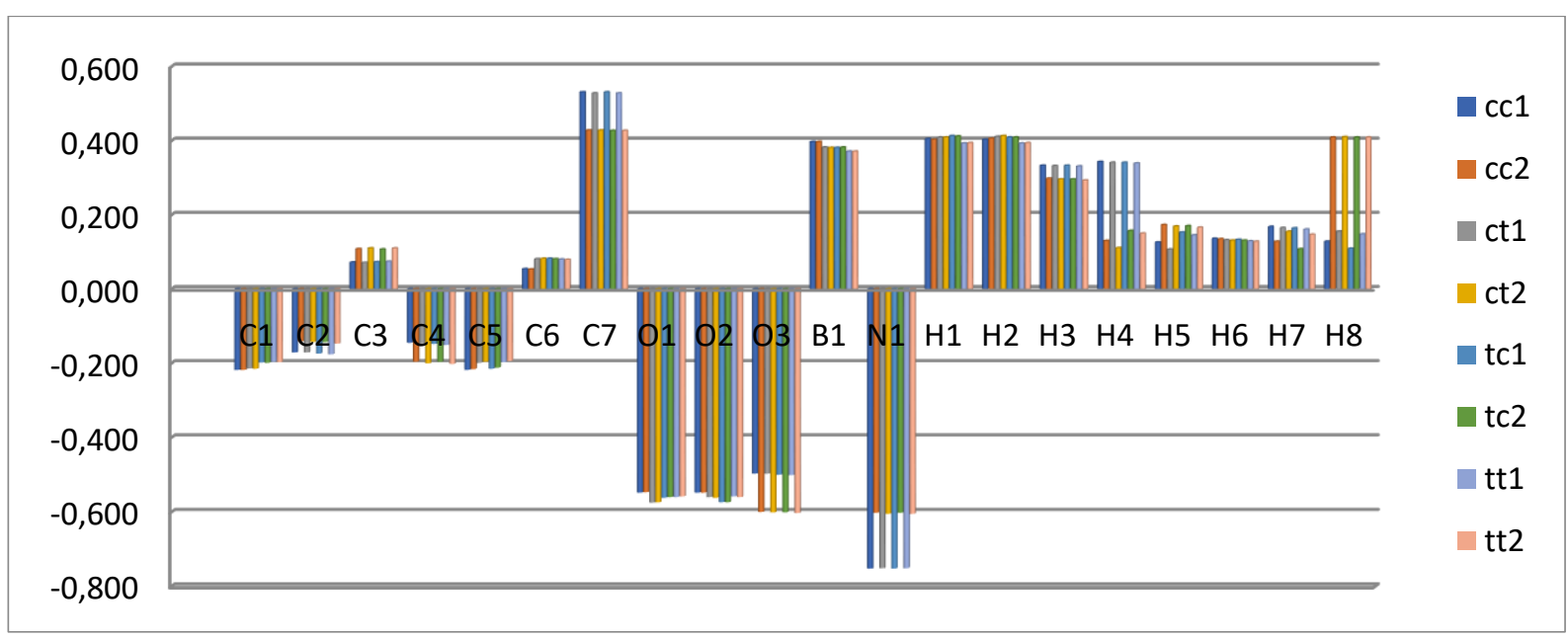

Figure 6. Mulliken Charge distribution of CAPBA

Table 4. Mulliken Charge distribution of CAPBA

\begin{tabular}{lrllllllr}
\hline Charges & CC1 & CC2 & CT1 & \multicolumn{1}{l}{ CT2 } & \multicolumn{1}{c}{ TC1 } & \multicolumn{1}{c}{ TC2 } & \multicolumn{1}{c}{ TT1 } & \multicolumn{1}{c}{ TT2 } \\
\hline C1 & -0.218 & -0.218 & -0.214 & -0.215 & -0.198 & -0.199 & -0.196 & -0.197 \\
C2 & -0.171 & -0.141 & -0.171 & -0.143 & -0.174 & -0.143 & -0.176 & -0.147 \\
C3 & 0.072 & 0.108 & 0.071 & 0.110 & 0.073 & 0.107 & 0.074 & 0.110 \\
C4 & -0.145 & -0.196 & -0.148 & -0.200 & -0.148 & -0.196 & -0.151 & -0.202 \\
C5 & -0.218 & -0.216 & -0.199 & -0.196 & -0.215 & -0.212 & -0.197 & -0.194 \\
C6 & 0.054 & 0.053 & 0.081 & 0.082 & 0.082 & 0.081 & 0.080 & 0.079 \\
C7 & 0.531 & 0.428 & 0.528 & 0.428 & 0.531 & 0.427 & 0.528 & 0.427 \\
O1 & -0.548 & -0.547 & -0.575 & -0.574 & -0.562 & -0.560 & -0.560 & -0.558 \\
O2 & -0.548 & -0.548 & -0.560 & -0.562 & -0.574 & -0.574 & -0.558 & -0.560 \\
O3 & -0.497 & -0.600 & -0.497 & -0.601 & -0.500 & -0.601 & -0.501 & -0.603 \\
B1 & 0.397 & 0.397 & 0.382 & 0.381 & 0.381 & 0.382 & 0.371 & 0.371 \\
N1 & -0.752 & -0.602 & -0.751 & -0.605 & -0.752 & -0.602 & -0.751 & -0.605 \\
H1 & 0.405 & 0.403 & 0.409 & 0.409 & 0.413 & 0.412 & 0.393 & 0.394 \\
H2 & 0.403 & 0.406 & 0.411 & 0.413 & 0.409 & 0.409 & 0.393 & 0.394 \\
H3 & 0.333 & 0.298 & 0.332 & 0.296 & 0.333 & 0.296 & 0.331 & 0.293 \\
H4 & 0.343 & 0.130 & 0.341 & 0.111 & 0.341 & 0.157 & 0.339 & 0.150 \\
H5 & 0.126 & 0.173 & 0.107 & 0.169 & 0.153 & 0.170 & 0.145 & 0.166 \\
H6 & 0.136 & 0.135 & 0.132 & 0.131 & 0.133 & 0.131 & 0.129 & 0.128 \\
H7 & 0.168 & 0.128 & 0.165 & 0.155 & 0.164 & 0.108 & 0.161 & 0.147 \\
H8 & 0.128 & 0.409 & 0.155 & 0.410 & 0.109 & 0.409 & 0.148 & 0.409 \\
\hline
\end{tabular}

\section{Molecular Orbitals analysis (HOMO-LUMO) and UV-Vis spectra}

HOMO (Highest Occupied Molecular Orbital) represents the highest energy level that at least one electron exists around the molecule. As just opposite LUMO (Lowest Unoccupied Molecular Orbital) represents the lowest energy level around the molecule in which there is no electron. The HOMO-LUMO gap is very important for charge transfers which affect the biological and chemical reactivities of the molecules. The molecules with small energy gaps 
are expected to be chemically reactive and called soft molecules. HOMO LUMO levels also determine the acidity or basicity of the molecules.

In the molecule, CAPBA HOMO-LUMO gap is $5.4 \mathrm{eV}$ which means this molecule can be supposed to be a "soft" molecule and of course the opposite is also true (figure 6.). The larger HOMO-LUMO gap always refers to higher kinetic stability and lower chemical reactivity. Molecule's hardness can be calculated via Eq. 3a and softness $(S)$ can be calculated via Eq $3 b$. $\eta=\left(\varepsilon_{\text {LUMO }}-\varepsilon_{\text {HOMO }}\right)$ Eq. 3a $S=1 / \eta$ Eq. $3 b$

While hard molecules which have a large gap between HOMO\&LUMO do not change their electron density easily, the soft molecules with a small gap of HOMO\&LUMO change their electron density relatively easily (Sahin et al., 2015, Pearson, 2005).

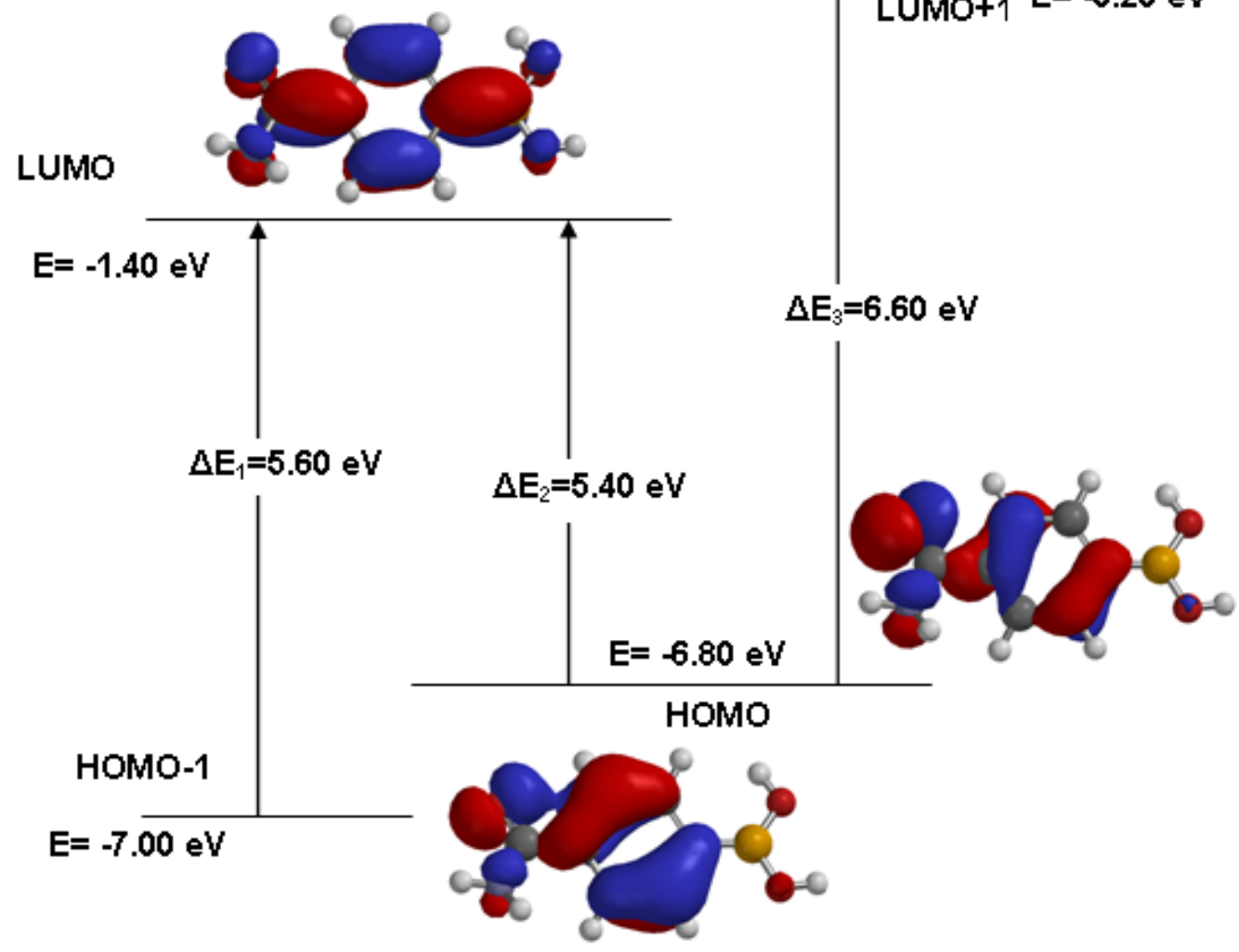

Figure 7. Electron transfers and corresponding $E$ values and HOMO-LUMO gap $\left(\Delta E_{2}\right)$ for TC form of CAPBA 
Table 5. The energy equivalencies for the transitions between conformers for the compound CAPBA

\begin{tabular}{|c|c|c|c|c|c|c|}
\hline Conformers & $\begin{array}{c}\text { Energy } \\
\text { (Hartree) }\end{array}$ & (Hartree) & $\begin{array}{l}\text { nergy Diff. } \\
\text { (kcalmol- } \\
{ }^{1} \text { ) }\end{array}$ & $(\mathrm{eV})$ & $\begin{array}{l}\text { Eq. Freq. } \\
\left(\mathrm{cm}^{-1}\right)\end{array}$ & $\begin{array}{c}\text { Dip. } \\
\text { Moment } \\
\text { (Debye) }\end{array}$ \\
\hline $\mathrm{CC}$ & - & 0.0052150 & 3.272463 & 0.141907 & 1144.56 & 3.57 \\
\hline CT & - & 0.0004960 & 0.311245 & 0.013497 & 108.86 & 4.09 \\
\hline $\mathrm{TC}$ & - & 0.0000000 & 0.00 & 0.00 & 0.00 & 2.29 \\
\hline TT & - & 0.0037000 & 2.321786 & 0.100682 & 812.06 & 5.24 \\
\hline
\end{tabular}

Table 6. MO energies and differences with $\lambda_{\max }$ values (simplified) for conformers of the compound CAPBA

\begin{tabular}{|c|c|c|c|c|c|c|c|c|c|c|}
\hline \multirow{2}{*}{ Conf. } & \multicolumn{2}{|l|}{ HOMO- } & \multirow{2}{*}{ LUMO } & \multirow{2}{*}{ LUMO+1 } & \multicolumn{3}{|c|}{$\Delta E(e V)$} & \multicolumn{3}{|c|}{$\lambda_{\max }$} \\
\hline & 1 & & & & $\Delta \mathrm{E}_{1}$ & $\Delta \mathrm{E}_{2}$ & $\Delta \mathrm{E}_{3}$ & & Calc. (Vac & \\
\hline CC & -7.3 & -6.9 & -1.5 & -0.6 & 5.8 & 5.4 & 6.3 & 213.77 & 229.60 & 196.80 \\
\hline CT & -7 & -6.8 & -1.4 & -0.3 & 5.6 & 5.4 & 6.5 & 221.40 & 229.60 & 190.74 \\
\hline TC & -7 & -6.8 & -1.4 & -0.2 & 5.6 & 5.4 & 6.6 & 221.40 & 229.60 & 187.85 \\
\hline TT & -6.8 & -6.6 & -1.2 & 0 & 5.6 & 5.4 & 6.6 & 221.40 & 229.60 & 187.85 \\
\hline
\end{tabular}

Table 7 Calculated Allowed electron transitions and corresponding $\lambda$ max values for CAPBA

\begin{tabular}{|c|c|c|c|c|c|c|c|c|c|}
\hline & \multirow{2}{*}{$\frac{\mathrm{nm}}{203.46}$} & \multirow{2}{*}{$\begin{array}{l}\text { Strength } \\
0.0046\end{array}$} & \multicolumn{2}{|c|}{$\begin{array}{l}\text { MO } \\
\text { Comp.\% }\end{array}$} & & \multirow{2}{*}{$\begin{array}{l}\mathrm{nm} \\
193.13\end{array}$} & \multirow{2}{*}{$\begin{array}{l}\text { Strength } \\
0.0039\end{array}$} & \multicolumn{2}{|c|}{$\begin{array}{r}\text { MO } \\
\text { Comp.\% }\end{array}$} \\
\hline & & & HOMO-4 -> LUMO & 66 & & & & HOMO-5 -> LUMO & 7 \\
\hline & & & HOMO-5 -> LUMO & 23 & & & & HOMO-3 -> LUMO+1 & 16 \\
\hline & 203.92 & 0.0794 & HOMO -> LUMO+1 & 34 & & 214.71 & 0.0616 & HOMO -> LUMO+1 & 58 \\
\hline & & & HOMO-3 -> LUMO+1 & 22 & & & & HOMO-1 -> LUMO+1 & 28 \\
\hline & & & HOMO-2 -> LUMO+1 & 20 & & 220.24 & 0.1069 & HOMO-3 -> LUMO & 55 \\
\hline & & & HOMO-1 -> LUMO & 14 & & & & HOMO $\rightarrow$ LUMO+1 & 13 \\
\hline \multirow[t]{14}{*}{ CC } & 206.77 & 0.037 & HOMO-3 -> LUMO & 76 & TC & & & HOMO $\rightarrow$ LUMO & 12 \\
\hline & 228.57 & 0.2988 & HOMO -> LUMO & 66 & & 222.74 & 0.2627 & HOMO-2 -> LUMO & 41 \\
\hline & & & HOMO-2 -> LUMO & 16 & & & & HOMO-3 -> LUMO & 32 \\
\hline & 239.69 & 0.0361 & HOMO-2 -> LUMO & 79 & & & & HOMO-1 -> LUMO & 12 \\
\hline & 249.07 & 0.0103 & HOMO-1 -> LUMO & 55 & & 250.42 & 0.0175 & HOMO-1 -> LUMO & 45 \\
\hline & & & HOMO $\rightarrow$ LUMO+1 & 33 & & & & HOMO-2 -> LUMO & 22 \\
\hline & & & & & & & & HOMO-2 -> LUMO+1 & 15 \\
\hline & & & & & & 284.98 & 0.0035 & HOMO -> LUMO & 70 \\
\hline & & & & & & & & HOMO-1 -> LUMO & 19 \\
\hline & 192.87 & 0.0024 & HOMO-5 -> LUMO & 88 & & 211.18 & 0.0292 & HOMO -> LUMO+1 & 61 \\
\hline & 200.45 & 0.081 & HOMO -> LUMO+1 & 42 & & & & HOMO-2 -> LUMO+1 & 26 \\
\hline & & & HOMO-3 -> LUMO+1 & 24 & & 219.6 & 0.0438 & HOMO-3 -> LUMO & 80 \\
\hline & & & HOMO-2 -> LUMO+1 & 15 & & 223.93 & 0.4045 & HOMO-2 -> LUMO & 61 \\
\hline & & & HOMO-1 -> LUMO & 13 & & 252.89 & 0.019 & HOMO-1 -> LUMO & 65 \\
\hline \multirow{7}{*}{ CT } & 208.03 & 0.0564 & HOMO-3 -> LUMO & 82 & TT & & & HOMO-2 -> LUMO+1 & 14 \\
\hline & 230.32 & 0.3379 & HOMO -> LUMO & 62 & 11 & 284.51 & 0.0036 & HOMO -> LUMO & 64 \\
\hline & & & HOMO-2 -> LUMO & 17 & & & & HOMO-2 -> LUMO & 23 \\
\hline & 240.37 & 0.0442 & HOMO-2 -> LUMO & 78 & & & & & \\
\hline & 251.23 & 0.0214 & HOMO-1 -> LUMO & 55 & & & & & \\
\hline & & & HOMO $\rightarrow$ LUMO+1 & 26 & & & & & \\
\hline & & & HOMO $\rightarrow$ LUMO & 13 & & & & & \\
\hline
\end{tabular}




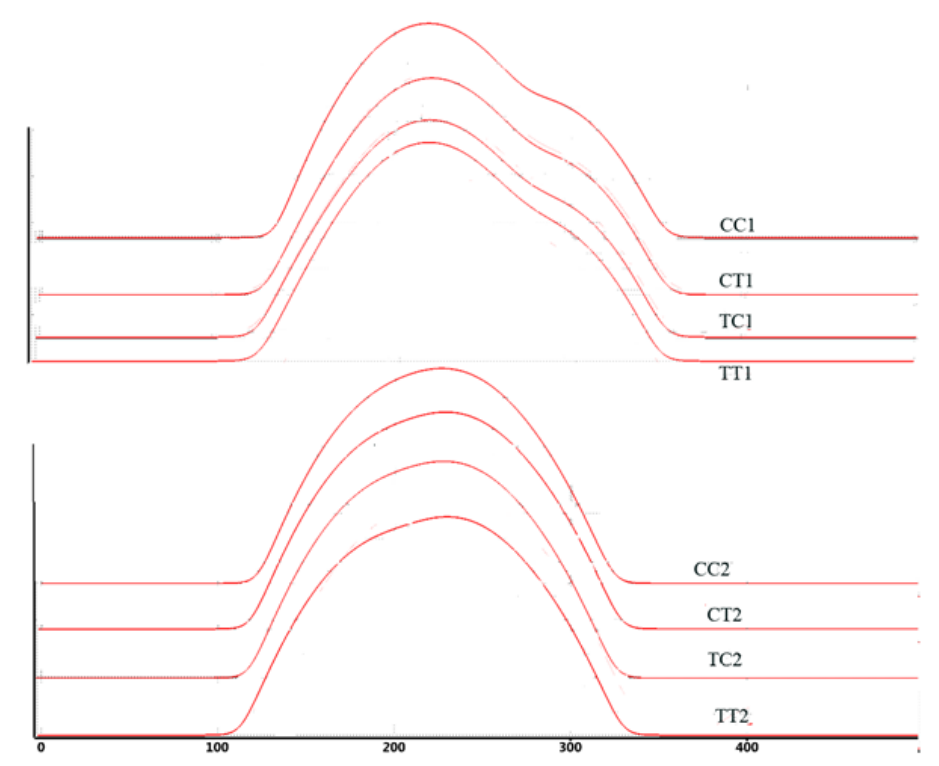

Figure 8. Calculated UV-Vis spectra for the compound CAPBA

\section{CONCLUSIONS}

In this study, the compound CAPBA was investigated for mainly its molecular structure using computational methods. Imported experimental values such as bond lengths and bond angles were compared to corresponding calculated ones (and were found to agree). Also, the molecule was surveyed for its UV-Vis spectra and HOMO\&LUMO analysis beside Mulliken charge distribution. The HOMO\&LUMO energy gap and UV-Vis spectra and also the hardness of the molecule were discussed. The obtained results were tabulated in corresponding sections and some results were depicted in graphs and figures. In calculations, the 6.31G* basis set was used under DFT's B3LYP level. The SPARTAN 14 quantum chemical calculation software was used in this study. The experimental and calculated results were compared in tables and verified each other.

The author declares that there is no Conflict of Interest with any person and/or institution.

\section{Supplementary material}

All requests from the readers who would like to obtain extra information such as pictures, tables, etc. will be responded with pleasure by the corresponding author. 


\section{REFERENCES}

Akalın R.,B., Ulusoy S., (2018), Boronik asit moleküllerinin Pseudomonas aeruginosa'da virülens faktörlerinin üretimine etkisi, Journal of Boron, 3 (3), 166 - 173

Apostolova M. D., Nikolova R. P., Shivachev B. L., (2010), (4-Carbamoylphenyl)boronic acid, Acta Crystallographica Section E Crystallographic Communications 66, 1273-1279.

Baker S.,J., Ding C.,Z., Akama T., Zhang Y.,K., Hernandez V., Xia Y., (2009) Therapeutic potential of boroncontaining compounds, Future Med Chem. 1(7), 1275-88.

Becke A.D., (1993), Density-functional thermochemistry. III. The role of exact exchange, Jounal of Chemical Physics 98, 5648-5652.

Beytur M., Yüksek H., (2018) 3-Fenil-4-(3-Sinnamoiloksibenzilidenamino)-4,5-Dihidro-1h-1,2,4-Triazol-5-On Molekülünün Spektroskopik Özellikleri, Caucasian Journal of Science, 5 (2), 65-80

Erdogdu Y., Güllüoğlu M., Kurt M., (2009), DFT, FT-Raman, FT-IR and NMR studies of 2-fluorophenylboronic acid, Journal of Raman Spectroscopy, 40(11), 1615-1623,

Hall D.G., (2006), Structure, properties, and preparation of boronic acid derivatives. Overview of their reactions and applications. Boronic acids: preparation and applications in organic synthesis and medicine, Wiley Online library.

Hehre, W., J., SPARTAN'14, (2014), Wavefunction Inc. Irvine CA, USA

Hehre W. J., (2014), "SPARTAN'14 Tutorial and User's Guide”, Wavefunction, Inc. Jensen F., (2017), Introduction to Computational Chemistry, Wiley

Kar A., (2003), Pharmacognosy and Pharmacobiotechnology, New Age International (P) Ltd., Publishers New Delhi

Karabacak M, Kose E, Atac A, Cipiloglu Ma, Kurt M., (2012), Molecular structure investigation and spectroscopic studies on 2,3-difluorophenylboronic acid: A combined experimental and theoretical analysis. Spectrochimica Acta - Part A: Molecular and Biomolecular Spectroscopy, 97, 892-908,

Kotan G., K., Yuksek H., (2019), Experimental (FT-IR, NMR) and Theoretical (B3PW91, B3LYP, HF) Analyses of 2(3-Ethyl-4,5-Dihydro-1H-1,2,4-Triazol-5-on-4-yl)-azomethine)-Benzoic Acide, Caucasian Journal of Science, 6 (1), 64-75

Lee C., Yang W., Parr R.G., (1998), Development of the Colle-Salvetti correlation-energy formula into a functional of the electron density, Physical Review B - Condensed Matter and Materials Physics 37 785-799

Lu, G.-P.; Voigtritter, K. R.; Cai, C.; Lipshutz, B. H., (2012), Ligand Effects on the Stereochemical Outcome of Suzuki-Miyaura Couplings. Journal Organic Chemistry, 77(8), 3700-3703,

Onishi T., (2018 ), "Quantum Computational Chemistry: Modelling and Calculation for Functional Materials", Springer-Singapore, pp. 49-53

Pearson R.G., (2005), Chemical hardness and density functional theory, Chemical Science Journal, 117(5), 369377 ,

Peter K., Vollhardt C., Schore N. E., (2011), Organic chemistry: structure and function, 6th ed. Freeman\&Comp. NY-US. 
Rani U, Karabacak M, Tanrıverdi O, Kurt M, Sundaraganesan N., (2012), The spectroscopic (FTIR, FT-Raman, NMR and UV), first-order hyperpolarizability and HOMO-LUMO analysis of methyl boronic acid. Spectrochimica Acta Part A: Molecular and Biomolecular Spectroscopy, 92(15), 67-77

Sachan Ak, Pathak Sk, Sinha L, Prasad O, Karabacak M, Asiri A.M., A, (2014), combined experimental and theoretical investigation of 2-Thienylboronic acid: Conformational search, molecular structure, NBO, NLO and FT-IR, FT-Raman, NMR and UV spectral analysis. Journal of Molecular Structucture, 1076, 639-650,

Şahin Z. S., Kaya Kantar G., Şaşmaz S., Büyükgüngör O., (2015), Synthesis, molecular structure, spectroscopic analysis, thermodynamic parameters and molecular modeling studies of (2methoxyphenyl)oxalate, Journal of Molecular Structucture, 1087, 104-112.

Silverstein R.M., Webster F.X., Kıemle D.J., (2005), Spectrometric Identification of Organic Compounds 7th Ed. John Wiley Sons INC.

Tjarks W., Anisuzzaman A.K., Liu L., Soloway S.H., Barth R.F., Perkins D.J., Adams D.M., (1992), Synthesis and in vitro evaluation of boronated uridine and glucose derivatives for boron neutron capture therapy. Journal Medicinal Chemistry, 35, 1628-1633

Turhan İrak Z., Beytur M., (2019), 4-Benzilidenamino-4,5-dihidro-1H-1,2,4-triazol-5-on Türevlerinin Antioksidan Aktivitelerinin Teorik Olarak İncelenmesi, Iğdır Üniversitesi Fen Bilimleri Enstitüsü Dergisi, 9(1): 512521

URL1.https://www.sigmaaldrich.com/catalog/product/aldrich/683876?lang=en\&region=TR (03.26.2020)

Zepeda-Velazquez L. C., (2015), Synthesis and characterization of novel stimuli-responsive silicone-boronic acid materials, (Unpublished doctoral dissertation), McMaster University Hamilton, Ontario.

Zheng H., Ghanbari S., Nakamura S., Hall D.G., (2012), Boronic Acid Catalysis as a Mild and Versatile Strategy for Direct Carbo- And Heterocyclizations of Free Allylic Alcohols, Angewandte Chemie Int. Ed., 51(25), 6187-6190,

Zheng H., Lejkowski M., Hall D.G., (2011), Mild and selective boronic acid-catalyzed 1,3-transposition of allylic alcohols and Meyer-Schuster rearrangement of propargylic alcohols, Chemical Science Journal, 2(7), 1305-1310. 